\title{
THE INDUCTION OF ATP ENERGIZED MITOCHONDRIAL VOLUME CHANGES BY CARCINOGENIC N-HYDROXY-N-ACETYL-AMINOFLUORENES WHEN COMBINED WITH SHOWDOMYCIN. A UNITARY HYPOTHESIS FOR CARCINOGENESIS $*, * *, * * * *$
}

\author{
Herbert I. Hadler, Barbra G. Daniel \\ and Raymond D. Pratt \\ Department of Chemistry, Southern Illinois University, \\ Carbondale, Illinois 62901, U.S.A.
}

(Received for publication February 1, 1971)

$\mathrm{N}-\mathrm{Hydroxy}-\mathrm{N}$-acetyl-2-aminofluorene, a well-known carcinogenic metabolite (Miller and Miller) derived from the carcinogen N-acetyl-2-aminofluorene induced an ATP energized mitochondrial volume change when combined with the thiol reagent showdomycin. Neither N-acetyl-2-aminofluorene nor showdomycin by themselves induced an ATP enegized mitochondrial volume change. Showdomycin could be replaced by other thiol reagents. The ATP energized mitochondrial volume change induced by gramicidin was partially inhibited by $\mathrm{N}$-hydroxy-N-acetyl-2-aminofluorene and the inhibition was relieved by showdomycin. N-Hydroxy-N-acetyl-2-aminofluorene inhibited mitochondrial respiration during a state of active phosphorylation. In accordance with the reasoning in our previous publications it was deduced that $\mathrm{N}$-hydroxy-N-acetyl-2-aminofluorene inhibited the cycle which meshed with the respiratory chain and exposed a strategically located pivotal mitochondrial thiol group.

The following data suggested a confluence of experimentation in oxidative phosphorylation and carcinogenesis. The parent carcinogen $\mathrm{N}$-acetyl-2-aminofluorene plus a thiol reagent did not induce an ATP energized mitochondrial volume change in keeping with a requirement for metabolic conversion of the parent carcinogen in order to engender carcinogenesis. N-Acetyl-3-aminofluorene (a marginal carcinogen in the rat as observed by Gutmans) did not induce an ATP energized mitochondrial volume change in combination with a thiol reagent. $\mathrm{N}$-Hydroxy-N-acetyl-3-aminofluorene which could be derived synthetically but not metabolically from the parent marginal carcinogen was carcinogenic (GUTMANN) and did induce an ATP energized mitochondrial volume change when combined with showdomycin.

* Supported by U.S.P.H.S. grant CA 10759 (National Cancer Institute). Mr. PratT was supported by a National Science Foundation Undergraduate Research Participation Program, grant GY-7637.

** A portion of this material was presented by H. I. HAdLer and B. G. DANiel on October 29, 1970, at the Sixth Midwest Regional American Chemical Society Meeting, Lincoln, Nebraska, U.S.A.

*** Abbreviations used. ATP: Adenosine-5'-triphosphate. ADP: Adenosine-5'-diphosphate. Pi: Inorganic phosphate. DNP: 2,4-Dinitrophenol. PHMB: $p$-Hydroxymercuribenzoate. pFCCP: Carbonyl cyanide $p$-trifluoromethoxyphenylhydrazone. NEMI: N-Ethylmaleimide. AAF: NAcetyl-2-aminofluorene. N-OH-AAF: N-Hydroxy-N-acetyl-2-aminofluorene. N-OAc-AAF : NAcetoxy-N-acetyl-2-aminofluorene. AAF-3: N-Acetyl-3-aminofluorene. N-OH-AAF-3: NHydroxy-N-acetyl-3-aminofluorene. Mw: Mitochondria. 
It was hypothesized that when the mitochondria were damaged, genetic material could be released from the mitochondria. The released genetic material could behave like an oncogenic virus and enter the nuclear genome. Thus carcinogenesis resulted from a disruption of the symbiotic relationship between the mitochondria and the host cell previously established during the course of evolution. In effect, carcinogenesis represented a partial reversal of evolution.

During the past several years, we have been using antibiotics and other exogenous agents of known structure to identify the functional groups of mitochondria involved in the related phenomena of oxidative phosphorylation, ion transfer and energized mitochondrial volume change, ${ }^{1 \sim 8)}$ Irrespective of the mechanism of energized mitochondrial volume changes, the occurrence of an ATP energized mitochondrial volume change dependent upon an agent or group of agents clearly indicated an interaction of the agent or group of agents with the mitochondria. As the exogenous agent or agents were of known structure, it was possible to recognize the nature of the mitochondrial functional groups which were involved.

It was deduced that a pivotal mitochondrial thiol group was strategically located between two catalytic cycles $^{6}$. One of the cycles meshed with the respiratory chain and the other cycle meshed with ATP, ADP, and Pi. The pivotal thiol group was exposed (that is, made reactive) either by the addition of an uncoupling agent such as $\mathrm{DNP}^{5)}$ or by the addition of a respiratory inhibitor such as antimycin ${ }^{4}$. When the exposed pivotal thiol group became conjugated with a thiol reagent such as the antibiotic showdomycin or the mercurial thiol reagent PHMB an ATP energized mitochondrial volume change was induced. ${ }^{4,5}$

The interaction of the nucleophilic terminal hydroxyl group of the antibiotic gramicidin with a non-phosphorylated electrophylic center located in the cycle which meshes with ATP, ADP and Pi had been previously inferred ${ }^{3)}$. It was shown that gramicidin was not inactivated when the terminal hydroxyl group was acetylated but was inactivated when the terminal hydroxyl group was methylated. Also when the gramicidin effect was energized by ATP, oligomycin was inhibitory ${ }^{1)}$ and when the gramicidin effect was energized by respiration oligomycin was not inhibitory while a low level of DNP (50 $\mu \mathrm{M})$ was inhibitory ${ }^{2}$.

These studies were extended to other well known agents of known structure which interacted with mitochondria ${ }^{8)}$. Dicoumarol and pFCCP mimicked DNP while the nonmercurial thiol reagent NEMI resembled showdomycin. The antibiotic valenomycin behaved like the antibiotic gramicidin. The concept of a strategically located pivotal mitochondrial thiol group thus rationalized the behavior of many exogenous agents and hence was of general significance. ${ }^{8)}$. The action of the lipophilic hydroxyquinone lapachol (an antitumor agent) resembled DNP. This observation was in keeping with our hypothesis for oxidative phosphorylation ${ }^{9)}$ which involved quinones, hydroxyquinones, and hydroquinones.

It has been unpopular for some time to link the phenomena of experimental carcinogenesis with either an impairment of respiration ${ }^{10,11)}$ or with damage to the 
mitochondria as suggested by WARBURG ${ }^{12)}$ many years ago. For some time research dealing with the mechanism of carcinogenesis has emphasized the binding of the carcinogen or one of its metabolite to either protein or nucleic acid ${ }^{13 \sim 30}$ ). The evaluation of binding to nucleic acids is currently under intensive investigation ${ }^{25)}$.

The thesis that binding to an intracellular macromolecule is an essential interaction leading to carcinogenesis does not rule out, however, other possible decisive interactions. Accordingly, we have examined on an empirical basis the possibility that target sites relevant to the process

Fig. 1. Structural formulae.

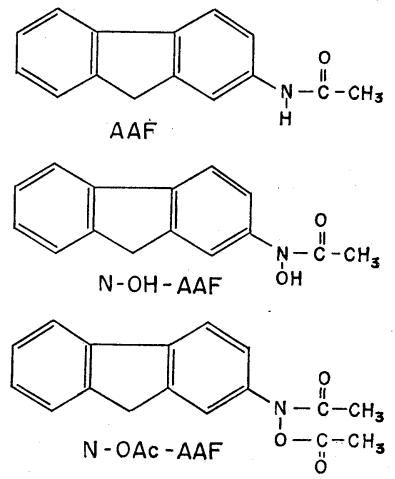
of carcinogenesis, exist in mitochondria. Our search for such an interaction was stimulated by our success with the antitumor agent lapachol7) as a similarity in properties of antitumor agents and carcinogens has of ten been noted ${ }^{31)}$. Lapachol is a vinylogue of a carboxylic acid and accordingly is an acidic molecule.

The structures of the many other agents (antimycin, DNP, dicoumarol, pFCGP, lawsone) which we have postulated to interact with the catalytic cycle which meshes with the respiratory chain also suggested that a compound with an acidic functional group could interact with this cycle. Much of the most recent ${ }^{25}$ ) exciting work in carcinogenesis has been done with the neutral carcinogenic amide AAF (Fig. 1) and with the hydroxamic acid N-OH-AAF (a carcinogenic metabolite of AAF (Fig. 1), and with acid anhydride derivatives of N-OH-AAF, e.g. N-OAc-AAF (Fig. 1). These considerations suggested that the hydroxamic acid N-OH-AAF might interact with the catalytic cycle which meshes with the respiratory chain. If such an interaction did indeed take place, then the interaction could be visualized as an ATP energized mitochondrial volume change on the further addition of a thiol reagent such as showdomycin.

It is the purpose of this report to present data showing that $\mathrm{N}-\mathrm{OH}-\mathrm{AAF}$ interacts with the catalytic cycle which meshes with the respiratory chain and that this interaction is relevant to the mechanism of carcinogenesis.

\section{Methods}

The procedures and methods have been previously described ${ }^{1,4,8)}$. The $\mathrm{pH}$ of the tris-chloride buffer is indicated on the diagrams. Incubations were at $27^{\circ} \mathrm{C}$ in standard rectangular quartz cuvettes with a $1-\mathrm{cm}$ path. The basic reaction mixture had a final volume of $3 \mathrm{ml}$ and contained $0.75 \mathrm{mg}$ of mitochondrial protein (prepared from rat liver); $333 \mu \mathrm{M}$ ATP (tris salt) which was added in $0.05 \mathrm{ml}$ by means of the adding-mixing device $^{2)}$ as indicated by an arrow on the diagrams; $75 \mathrm{~mm}$ sucrose; and $75 \mathrm{~mm}$ trischloride buffer. A decrease in absorbancy at $520 \mathrm{~nm}$ was considered to be a measure of mitochondrial swelling. A Model 2000 automatic spectrophotometer manufactured by Gilford Instrument Laboratories, Incorporated, Oberlin, Ohio, was used. All cations were added in the form of chloride salts and all anions were added in the form of tris salts, neutralized to $\mathrm{pH}$ 7.4. Solutions of AAF, AAF-3, N-OH-AAF and N-OH-AAF-3 were prepared in $95 \%$ ethanol (distilled) and added to the incubation mixtures in a volume of $0.06 \mathrm{ml}$. All controls contained the appropriate amount of alcohol. The figures and legends provide further experimental details. 
Oxygen consumption was measured in an oxygen monitor which utilized a Clark electrode. The instrument (Model 53SA) was manufactured by Yellow Springs Instrument Company, Yellow Springs, Ohio. A $10^{\prime \prime}$ recorder, Model 1005, manufactured by Beckman Instruments, Fullerton, California was used in conjunction with the oxygen monitor.

Commercial gramicidin, obtained from Mann Research Laboratories, Inc. (New York, N.Y., U.S.A.) was used. The activity of such a mixture of gramicidins had been previously shown to be indistinguishable from gramicidin $\mathrm{A}^{3)}$. The molecular weight of the mixture was arbitrarily taken as 1,870 . We wish to thank Dr. Ken'Ichi TAKedA of Shionogi and Company, Osaka, Japan for the showdomycin used in this work. Dr. Helmut Gutmann (Minneapolis, Minn., U.S.A.) generously provided samples of AAF-3 and NOH-AAF-3 and Dr. Elizabeth Weisburger (Washington D.C., U.S.A.) kindly provided $\mathrm{N}-\mathrm{OH}-\mathrm{AAF}$. AAF was purchased from Mann Research Laboratories (New York, N. Y., U.S.A.), and crystallized four times from ethanol and water.

\section{Results}

It is seen in Fig. 2 that the combination of $300 \mu \mathrm{M}$ showdomycin with $300 \mu \mathrm{M}$ $\mathrm{N}-\mathrm{OH}-\mathrm{AAF}$ induced an ATP energized mitochondrial volume change at $\mathrm{pH}$ 7.4. The phenomena was inhibited by the antibiotic oligomycin and thus was related to oxidative phosphorylation. ${ }^{32}$ The enhancement of the phenomena by graded increases in the concentration of $\mathrm{N}-\mathrm{OH}-\mathrm{AAF}$ is shown in Fig. 3. In Fig. 4, the enhancement of the phenomena by increasing the $\mathrm{pH}$ from 7.4 to 7.8 is shown and thus the data supports the involvement of a ionizable nucleophilic thiol group. The addition of $2 \mathrm{~mm}$ potassium ions had a minimal effect (Fig. 5). The addition of $2 \mathrm{~mm}$ malate ions increased the period of the oscillation in a characteristic empirical manner (Fig. 5) observed in other experiments. ${ }^{6,7)}$ The similar effectiveness of $300 \mu \mathrm{M}$ of the thiol reagent ${ }^{33)}$ showdomycin with appropriate amounts of other thiol reagents viz., $30 \mu \mathrm{M}$ NEMI and $3 \mu \mathrm{M}$ PHMB was observed (Fig. 6). Again, with all three thiol

Fig. 2. N-OH-AAF combined with showdomycin and effect of oligomycin.

Basic medium, see methods.

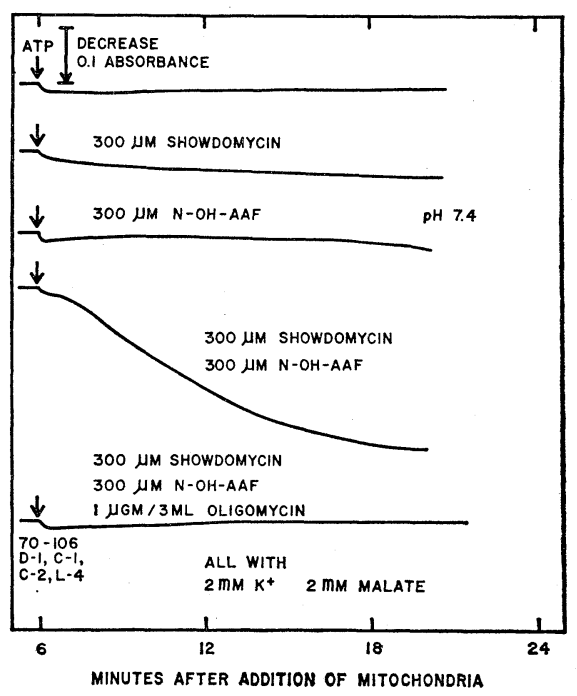

Fig. 3. Various concentrations of $\mathrm{N}-\mathrm{OH}-\mathrm{AAF}$.

Basic medium, see methods.

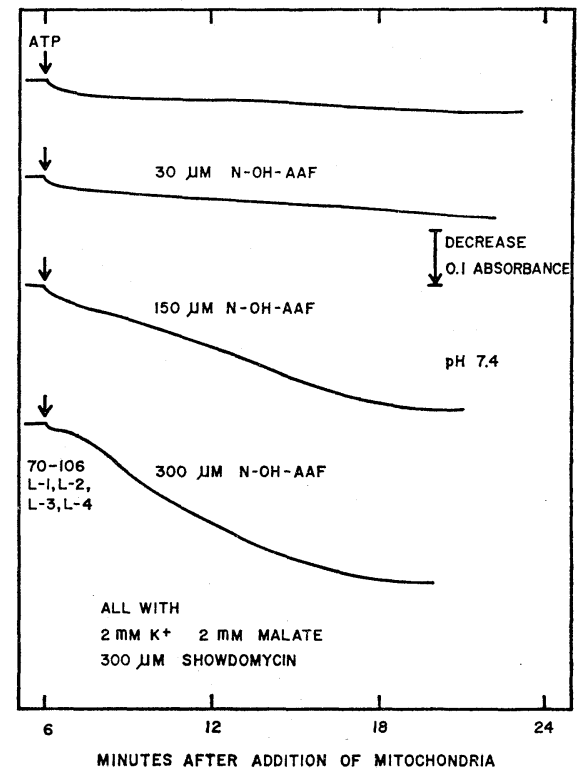


Fig. 4. Effect of $\mathrm{pH}$.

Basic medium, see methods.

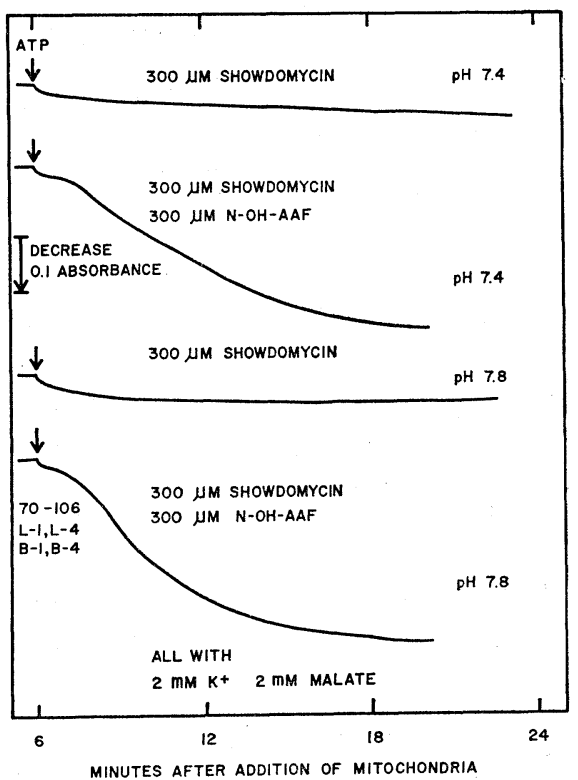

Fig. 5. Role of potassium ion and L malate.

Basic medium, see methods.

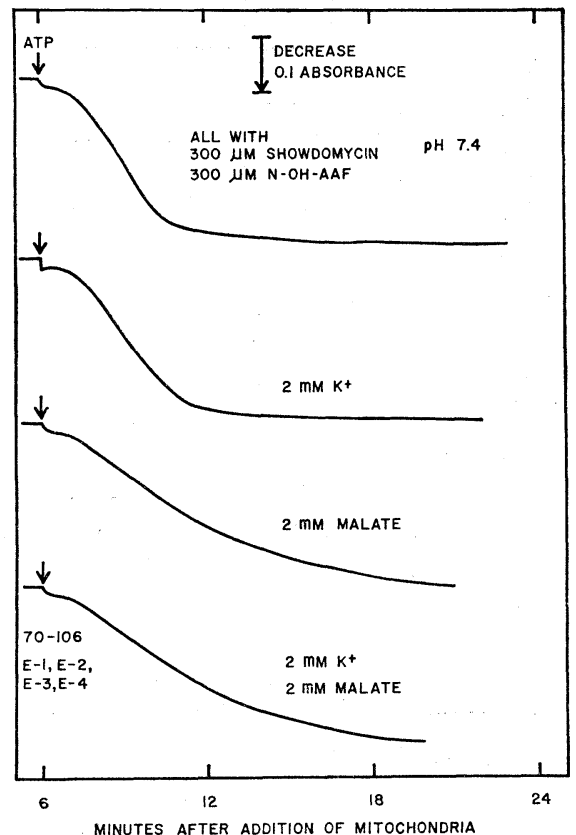

Fig. 6. Various thiol reagents.

Basic medium, see methods.

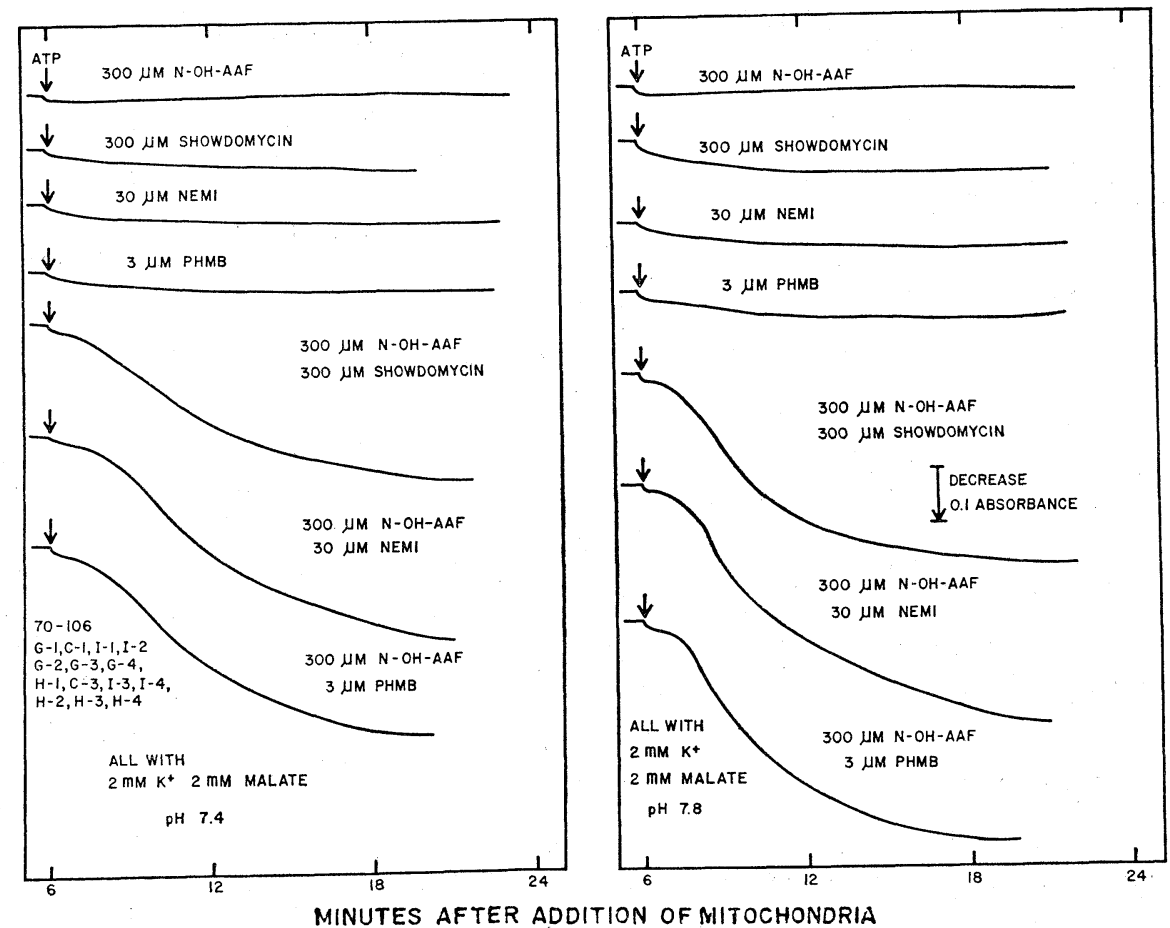


Fig. 7. AAF combined with various thiol reagents. Basic medium, see methods.

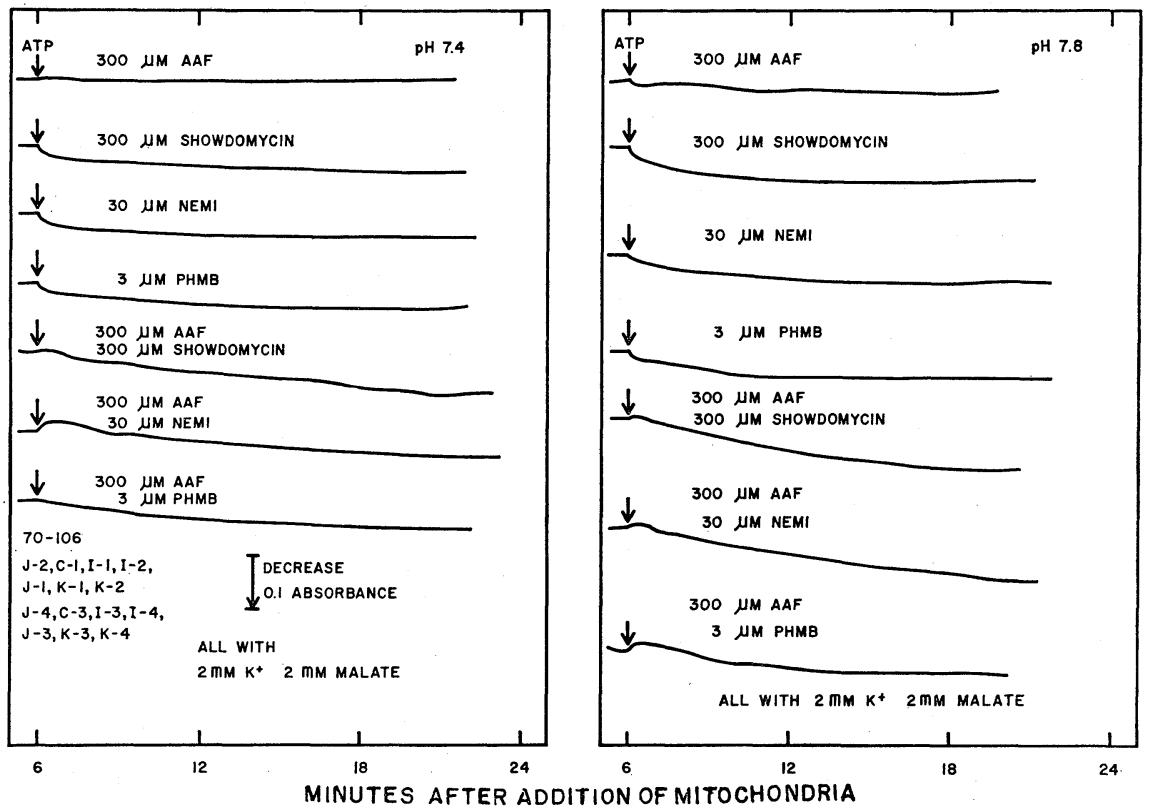

reagents the phenomena was enhanced when the $\mathrm{pH}$ was raised from 7.4 to 7.8 . In Fig. 7 the ineffectiveness of the carcinogen AAF when combined with the thiol reagents showdomycin, NEMI, and PHMB is observed at $\mathrm{pH}$ 7.4 and 7.8. It is seen in Fig. 8 that the ATP energized mitochondrial volume change induced by the antibiotic gramicidin in combination with the permeant ions potassium and malate was partially inhibited by the addition of 300 $\mu \mathrm{M} \mathrm{N}-\mathrm{OH}-\mathrm{AAF}$. The inhibition was relieved by the further addition of showdomycin to the combination of gramicidin plus $\mathrm{N}-\mathrm{OH}-\mathrm{AAF}$ (Fig. 9). In all the responsive systems containing gramicidin (Fig. 10) i.e., gramicidin alone, gramicidin plus $\mathrm{N}-\mathrm{OH}-\mathrm{AAF}$ plus showdomycin, and gramicidin plus showdomycin, the addition of potassium ion was a requirement or had a decided effect in enhancing the phenomena. This is in agreement with our previous observations ${ }^{6,7)}$ and it follows that the gramicidin system remained intact in the relieved system of gramicidin plus N-OH-AAF plus showdomycin. It is also seen that the addition of
Fig. 8. Inhibition of gramicidin system by $\mathrm{N}-\mathrm{OH}-\mathrm{AAF}$. Basic medium. see methods.

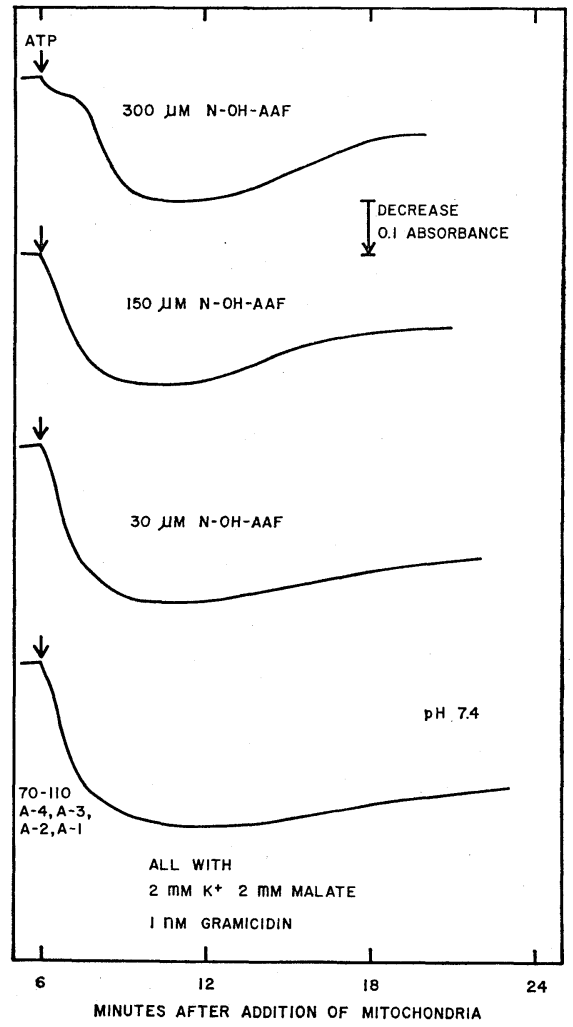


Fig. 9. Showdomycin plus the inhibited system. Basic medium, see methods.

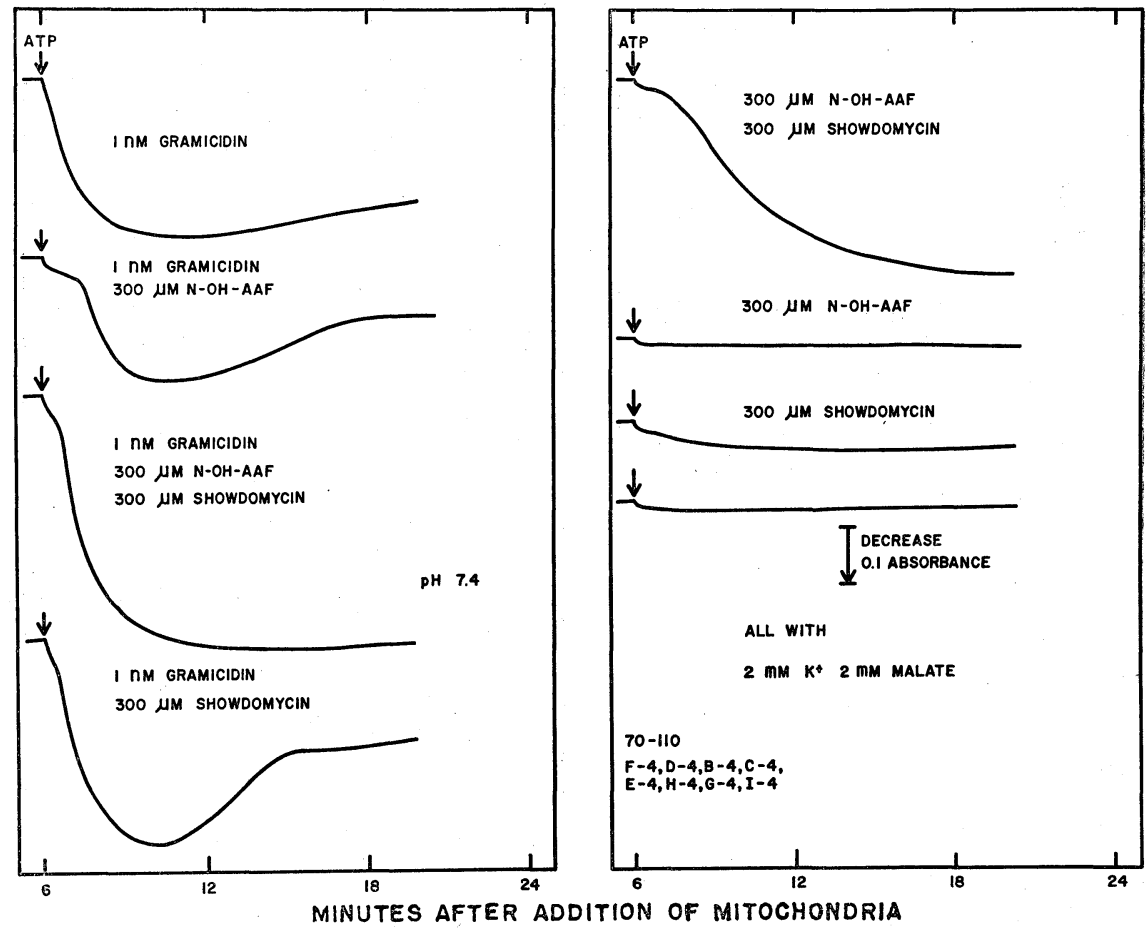

malate to the relieved system which contained showdomycin (that is, N-OH-AAF plus showdomycin plus gramicidin) caused an increase in the amplitude of oscillation. This increase is characteristic of active systems dependent upon showdomycin. ${ }^{6,7)}$ It is seen in Fig. 11 that AAF-3 when combined with showdomycin was inactive while $\mathrm{N}-\mathrm{OH}-\mathrm{AAF}-3$ when combined with showdomycin was active in inducing an ATP energized mitochondrial volume change and the phenomenon was enhanced when the $\mathrm{pH}$ was raised from 7.4 to 7.8 .

The inhibition of respiration by $\mathrm{N}-\mathrm{OH}-\mathrm{AAF}$ of an active coupled mitochondrial system, is clearly shown in Fig. 12.

\section{Discussion}

$\mathrm{N}-\mathrm{OH}-\mathrm{AAF}$ when combined with one of the reagents viz, showdomycin, NEMI or PHMB induced an ATP energized mitochondrial volume change. The effect was enhanced by raising the $\mathrm{pH}$ from 7.4 to 7.8 . The ability of showdomycin ${ }^{33)}$ and the other thiol reagents to conjugate with thiols and the effect of raising the $\mathrm{pH}$ implicated the exposure of an ionizable mitochondrial thiol group by $\mathrm{N}-\mathrm{OH}-\mathrm{AAF}$. The volume change data did not determine whether $\mathrm{N}-\mathrm{OH}-\mathrm{AAF}$ was an uncoupling agent such as DNP or a respiratory inhibitor such as antimycin. The experiment with the oxygen electrode established $\mathrm{N}$ $\mathrm{OH}-\mathrm{AAF}$ as a respiratory inhibitor.

In accordance with our previous rationalizations regarding the respiratory inhibitors rotenone $^{5)}$ and antimycin $\left.{ }^{4}\right) \mathrm{N}-\mathrm{OH}-\mathrm{AAF}$ interacted with the cycle which meshed with the respiratory chain. Because of this interaction a strategically located mitochondrial thiol group became unable to conjugate with its normal electrophilic acceptor located in the cycle which meshed with the respiratory chain. The thiol group thus became exposed 
Fig. 10. Role of ions in the reinstated system.

Basic medium, see methods.

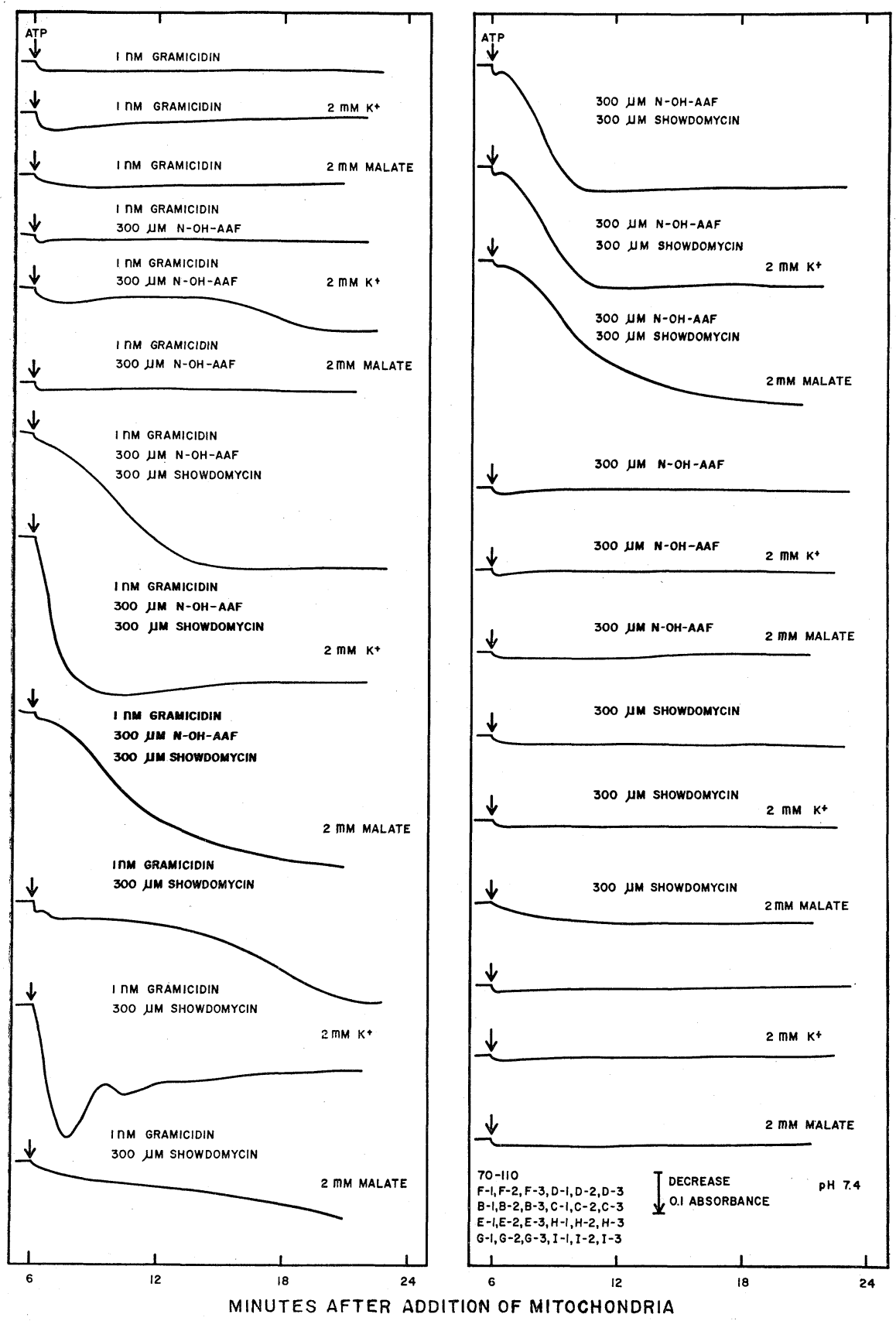

i.e. capable of conjugating with an exogenous thiol reagent such as showdomycin, NEMI, or PHMB. This conjugation resulted in an ATP energized mitochondrial volume change to be induced by the combination of $\mathrm{N}-\mathrm{OH}-\mathrm{AAF}$ plus a thiol reagent.

The pivotal nature of the strategically located mitochondrial thiol group was revealed as follows: Firstly, the exposed nucleophilic thiol group was able to compete with the nucleophilic hydroxyl of gramicidin for the nonphosphorylated electrophilic center in the cycle which meshed with ATP, ADP and.Pi. As a result of this competition, the ATP 
Fig. 11. AAF-3 and N-OH-AAF-3 combined with showdomycin.

Basic medium, see methods.

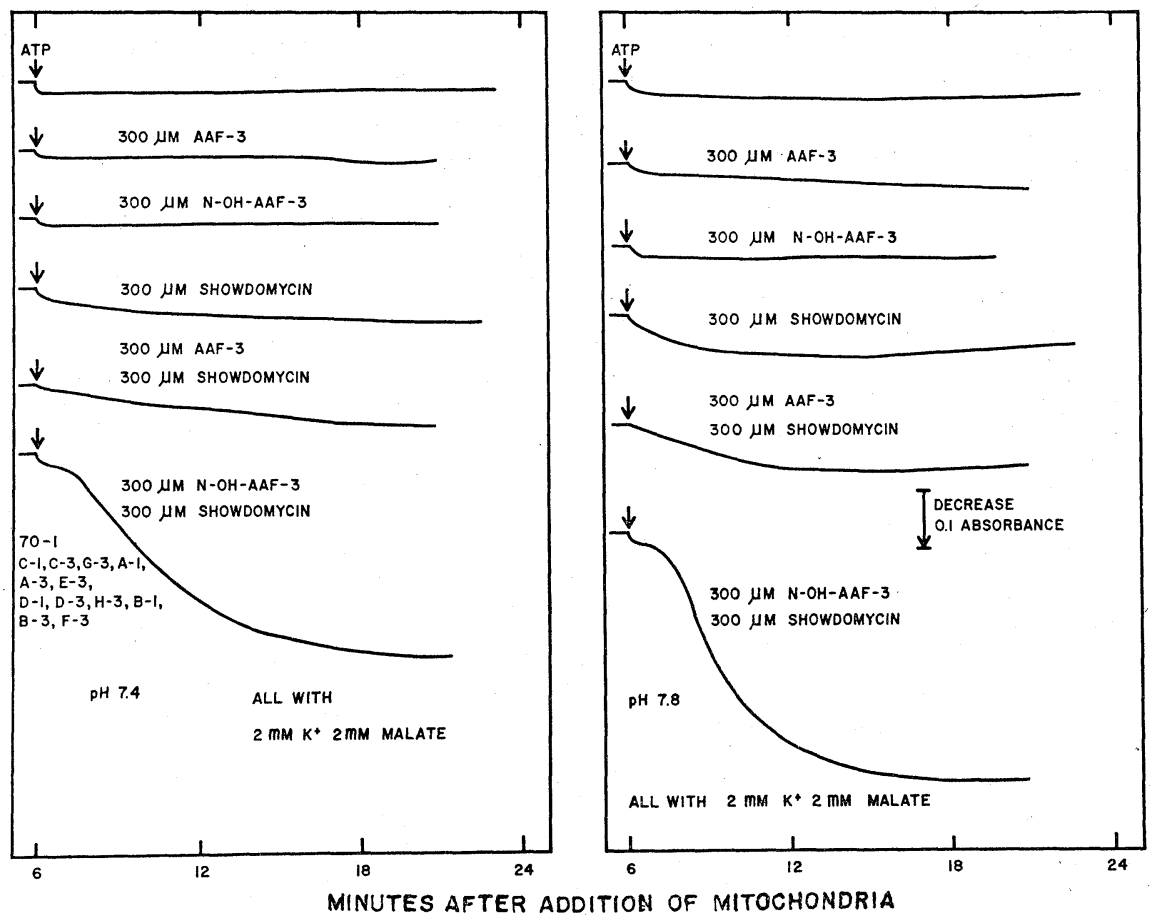

energized mitochondrial volume change induced by gramicidin was partially inhibited by $\mathrm{N}-\mathrm{OH}-\mathrm{AAF}$. Secondly, the partial inhibition of the gramicidin system was relieved by adding showdomycin which in our explanation essentially tied up and thus eliminated the exposed competitive thiol group responsible for the partial inhibition. The pivotal thiol group thus was able to communicate with the cycle which meshed with the respiratory chain and the cycle which meshed with ATP, ADP, and $\mathrm{Pi}^{6}$ ).

The magnitude of the mitochondrial volume change induced by the combination of N-OH-AAF plus showdomycin closely resembled that previously reported for the combination of antimycin plus showdomycin..$^{4,5)}$ It is likely that $\mathrm{N}-\mathrm{OH}-\mathrm{AAF}$ inhibits the second coupling site of the respiratory chain. The volume change data does not however reveal this information. Other types of future experiments will be necessary to determine the precise locale of the respiratory chain which is inhibited by $\mathrm{N}-\mathrm{OH}-\mathrm{AAF}$.

The carcinogen AAF, unlike its carcinogenic metabolite $\mathrm{N}-\mathrm{OH}-\mathrm{AAF}^{34 \sim 37)}$ did not induce a significant ATP energized mitochondrial volume change when combined with any of the thiol reagents showdomycin, or NEMI, or PHMB.

The in vivo carcinogenic and N-hydroxylation ${ }^{34 \sim 37)}$ data and the in vitro mitochondrial volume change data may be related as follows: When the carcinogen AAF is
Fig. 12. Effect of N-OH-AAF on mitochondrial respiration.

The total volume of the system was $3.0 \mathrm{ml}$. The final concen trations were sucrose $0.25 \mathrm{M}$; $\mathrm{KCl} 20 \mathrm{~mm} ; \mathrm{MgCl}_{2} 5 \mathrm{mM}$; potassium phosphate buffer ( $\mathrm{pH} 7.2$ ), $10 \mathrm{~mm}$; Tris chloride (pH 7.4), $20 \mathrm{~mm} ; \beta$-hydroxybutyric : acid $13 \mathrm{~mm}$; $\mathrm{N}-\mathrm{OH}-\mathrm{AAF}$ was added in $0.1 \mathrm{ml} 95 \%$ ethanol. The $95 \%$ ethanol by itself did not effect the rate of respiration in other control experiments. There were $5 \mathrm{mg}$ of mitochondrial protein in the $3 \mathrm{ml}$ system.

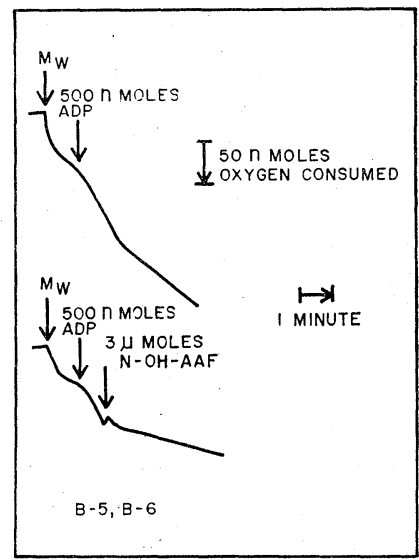


fed to a rat, the carcinogen becomes hydroxylated at the endoplasmic reticulum of a liver cell (as has been observed by others in vitro). ${ }^{38)}$ The generated $\mathrm{N}-\mathrm{OH}-\mathrm{AAF}$ then leaves the endoplasmic reticulum, interacts with the mitochondria and exposes the strategically located pivotal mitochondrial thiol group. A mechanistic requirement for $\mathrm{N}$-hydroxylation is thus established. These data point to a useful experimental confluence of the fields of oxidative phosphorylation and carcinogenesis.

The observations with AAF-3 and N-OH-AAF-3 provided further support for the experimental confluence of carcinogenesis with oxidative phosphorylation. Gutmann et al..$^{39}$ observed that AAF-3 was a marginal carcinogen in the rat and was not converted by the rat in vivo to $\mathrm{N}-\mathrm{OH}-\mathrm{AAF}-3$. When $\mathrm{N}-\mathrm{OH}-\mathrm{AAF}-3$ was prepared synthetically, the synthesized hydroxamic acid proved to be carcinogenic in the rat. ${ }^{39)}$ Accordingly, $\mathrm{N}-\mathrm{OH}-$ AAF-3 like N-OH-AAF should interact with mitochondria and expose the strategically located pivotal mitochondrial thiol group while AAF-3 should not interact with the mitochondria in this manner. It was observed that AAF-3 in combination with showdomycin did not induce an ATP energized mitochondrial volume change while $\mathrm{N}-\mathrm{OH}-\mathrm{AAF}-3$ in combination with the thiol reagent showdomycin or NEMI or PHMB did induce an ATP energized mitochondrial volume change. Thus the in vitro mitochondrial data are in agreement with the in vivo carcinogenic and metabolic data from Gutmann's laboratory.

These observations do suggest a relationship between an interference with the flux of energy through the mitochondria and chemical carcinogenesis. There are reports which have focused attention on changes in mitochondrial structure as a consequence of chemical carcinogenesis. Clerici and CudKovicz ${ }^{40)}$ fed the carcinogenic azo dye 4-dimethylaminazobenzene and observed by means of electron microscopy an increased rigidity in rat liver mitochondria. Eмmeloт and Bos $^{41)}$ fed the carcinogen 4-monomethylaminoazobenzene for five months to rats and observed that the swelling of rat liver mitochondria induced by thyroxine was reduced. Arcos and coworkers ${ }^{42,43}$ also used mitochondrial swelling as an index of structural alteration in the mitochondria during chemical carcinogenesis. They observed drastic oscillatory changes in rat liver mitochondria when they fed carcinogenic azo dyes, AAF, and ethionine. Non carcinogens did not produce drastic effects on the mitochondrial swelling index. These workers also observed a decided decrease in the mitochondrial content (as measured by mitochondrial protein) of rat liver during azo dye carcinogenesis $^{42)}$ and thus confirmed the earlier work of PRICE et al. ${ }^{44,45)}$ and StrIEBICH et al. ${ }^{46)}$ Other investigators drew the broad conclusion that neoplastic cells have less mitochondria than normal cells. ${ }^{11,47 \sim 50)}$

There are therefore several pieces of evidence linking an alteration of mitochondrial function or structure with carcinogenesis. Nevertheless, in view of current research which associates changes in the genome with viral carcinogenesis ${ }^{51,52)}$ the thesis that an alteration of mitochondrial machinery or structure has a causal relationship to carcinogenesis is not very satisfying. Accordingly, the following hypothesis which relates a disturbance in oxidative phosphorylation to nuclear genetic alteration and carcinogenesis is proposed.

Many investigators agree that during the course of evolution mitochondria was a procaryotic cell which invaded a host cell. As a consequence of evolution the mitochondria and the host cell developed a symbiotic existence with each other. (There are several recent excellent reviews on this subject. $)^{53,54,55)}$ We have noted with interest that the mitochondria is an organelle with its own relatively simple unique compliment of DNA and RNA. It is thus possible that the mitochondrial genetic material which is normally separated by a membrane system from the remainder of the cell may not necessarily be compatible with the normal genetic material in the nucleus of the cell. It is also known that when an appropriate exogenous oncogenic virus invades a host cell and alters the nuclear genome, ${ }^{51,52)}$ cancer develops.

We do hypothesize that when the mitochondrial machinery for oxidative phosphorylation is disturbed in a subtle fashion by an agent which is not present in adequate 
concentration to kill the cell, the mitochondrial membrane is altered. It then becomes possible for genetic material to leak through the mitocondrial membrane (in perhaps a single mitochondrion) and act like an exogenous oncogenic virus which has penetrated the cellular membrane. The mitochondrial genetic material may thus eventually alter the genome of the cell. In this way, a cancerous cell may be generated. Essentially the symbiotic relationship that existed between mitochondria and the host cell has been disrupted and evolution has been partially reversed. In this suggested scheme the chemical carcinogen engendered a process which released a latent oncogenic virus which was part of the normal genetic makeup of the mitochondria.

There also could be other means of damaging the membrane of the mitochondria (e.g. radiation injury or heat). Our hypothesis is accordingly a unitary hypothesis for carcinogenesis. Perhaps future research will implicate the release of other oncogenic genetic material from other organelles.

We are currently examining other classes of carcinogens and plan to report that appropriate metabolites of other classes of carcinogens also interfere with the normal flux of energy in the mitochondria.

The energized mitochondrial volume change phenomena might eventually become a useful rapid in vitro test which can help detect environmental carcinogenic hazards. Basic principles and generalizations are necessary to begin to cope with the potential biological hazards of the multitude of new compounds which have become part of the modern environment.

\section{References}

1) Falcone, A. B. \& H. I. Hadler: Action of gramicidin on mitochondria. I. Ion-dependent mitochondrial volume changes energized by adenosine-5'-triphosphate. Arch. Biochem. Biophys. $124: 91 \sim 109,1968$

2) Hadler, H. I. \& A. B. Falcone: Action of gramicidin on mitochondria. II. Ion-dependent mitochondrial volume changes energized by substrate oxidation. Arch. Biochem. Biophys. $124: 110 \sim 114,1968$

3) Falcone, A. B. \& H. I. Hadler: Action of gramicidin on mitochondria. III. Activity of gramicidin derivatives. Arch. Biochem. Biophys. $124: 115 \sim 121,1968$

4) Hadler, H. I.; B. E. Claybourn \& T. P. Tschang: Mitochondrial volume changes induced by the antibiotic showdomycin. Biochem. Biophys. Res. Commun. $31: 25 \sim 31,1968$

5) Hadler, H. I.; B. E. Claybourn \& T. P. Tschang: The mode of action of dinitrophenol revealed by mitochondrial volume changes requiring rotenone or antimycin or dinitrophenol with showdomycin. J. Antibiotics $21: 575 \sim 581,1968$

6) Hadler, H. I.; B. E. Claybourn, T. P. Tchang \& T. L. Moreau: The pivotal position of the mitochondrial thiol group exposed by dinitrophenol located by means of ATP energized mitochondrial volume changes requiring gramicidin, showdomycin, and dinitrophenol. J. Antibiotics $22: 183 \sim 188,1969$

7) Hadler, H. I. \& T. L. Moreau: The induction of ATP energized mitochondrial volume changes by the combination of the two antitumor agents showdomycin and lapachol. J. Antibiotics $22: 513 \sim 520,1969$

8) Hadler, H. I. ; B. E. Claybourn \& T. P. Tschang: Combinations of agents which induce ATP energized mitochondrial volume changes. J. Antibiotics $23: 276 \sim 287,1970$

9) HaDLER, H. I.: An hypothesis for oxidative phosphorylation. Experientia $17: 268 \sim 269,1961$

10) LePage, G. A. \& F. J. Henderson: Biochemistry of tumors. Progress in Experimental Tumor Research 1: 440 476, 1960

11) Wenner, C. B.: Progress in tumor enzymology. Advances in Enzymology 29 : 321 390, 1967

12) Warburg, O.: The metabolism of tumors. R. R. Smith, New York, 1931.

13) Miller, E. C. \& J. A. Miller: The presence and significance of bound aminoazo dyes in the livers of rats fed $p$-dimethylaminoazobenzene. Cancer Research $7: 468 \sim 480,1947$

14) Miller, E. C. \& J. A. Miller: In vivo combinations between carcinogens and tissue constituents and their possible role in carcinogenesis. Cancer Research $12: 547 \sim 556,1952$ 
15) Heidelberger, C: \& M. G. Moldenhauer: The interaction of carcinogenic hydrocarbons with tissue constituents. IV. A quantitative study of the binding to skin proteins of several ${ }^{14} \mathrm{C}-$ labeled hydrocarbons. Cancer Research $16: 442 \sim 449,1956$

16) Brookes, P. \& P. D. LAWLEY: Evidence for the binding of polynuclear aromatic hydrocarbons to the nucleic acids of mouse skin. Relationship between carcinogenic power of hydrocarbons and their binding to deoxyribonucleic acid. Nature $202: 781 \sim 784,1964$

17) Marroquin, F. \& E. Farber: The binding of 2-acetylaminofluorene to rat liver ribonucleic acid in vivo. Cancer Research $25: 1262 \sim 1269,1965$

18) Brookes, P.: Quantitative aspects of the reaction of some carcinogens with nucleic acids and the possible significance of such reactions in the process of carcinogenesis. Cancer Research $26: 1994 \sim 2003,1966$

19) Miller, E. C. \& J. A. Miller: Mechanism of chemical carcinogenesis: Nature of proximate carcinogens and interactions with macromolecules. Pharmacol. Rev. $18: 805 \sim 838,1966$

20) Dingman, C. W. \& M. B. Sporn: The binding of metabolites of aminoazo dyes to rat liver in vivo. Cancer Research $27: 938 \sim 944,1967$

21) Warwick, G. P. \& J. J. Roberts: Persistent binding of butter yellow metabolites to rat liver DNA. Nature $213: 1206 \sim 1207,1967$

22) Goshman, L. M. \& C. Heidelberger: Binding of tritium labelled polycyclic hydrocarbons to DNA of mouse skin. Cancer Research $27: 1678 \sim 1688,1967$

23) Swann, P. F. \& P. N. MaGeE: Nitrosamine-induced carcinogenesis. The alkylation of nucleic acids of the rat by $\mathrm{N}$-methyl-N-nitrosourea, dimethylnitrosoamine, dimethylsulphate, and methyl methanesulfonate. Biochem. J. $110: 39 \sim 47,1968$

24) Szafarz, D. \& J. H. Weisburger: Stability of binding of label from N-hydroxy-N-2-fluorenylacetamide to intracellular targets, particularly deoxyribonucleic acid in rat liver. Cancer Research 29 : 962 968, 1969

25) Miller, J. A.: Carcinogenesis by chemicals: An overview-G.H.A. Clowes memorial lecture. Cancer Research $30: 559 \sim 576,1970$

26) DeBaun, J. R.; E. C. Miller \& J. A. Miller: N-Hydroxy-2-acetylaminofluorene sulfotransferase: Its probable role in carcinogenesis and in protein-(methion-s-yl)-binding in rat liver. Cancer Research $30: 577 \sim 595,1970$

27) Maher, Sr. V. M.; J. A. Miller, E. C. Miller \& W. C. Summers: Mutations and loss of transforming activity of Bacillus subtilis DNA after reaction with esters of carcinogenic N-hydroxy aromatic amides. Cancer Research $30: 1473 \sim 1480,1970$

28) Heidelberger, C.: Chemical carcinogenesis, chemotherapy. Cancer's continuing core challengesG.H.A. Clowes memorial lecture. Cancer Research $30: 1549 \sim 1569,1970$

29) Grunberg, D. \& I. B. Weinstein: Effect of 2-acetylaminofluorene (AAF) binding to guanosine on coding properties of ribopolynucleotides and triplets. Abstracts Tenth International Cancer Congress, May 22 29, 1970, Houston, Texas (U.S.A.). Abstract number 613, page 378

30) Lijinsky, W.; K. Y. Lee \& C. H. Gallagher: Interaction of aflatoxins $b_{1}$ and $g_{1}$ with tissues of the rat. Cancer Research $30: 2280 \sim 2283,1970$

31) Clayson, D. B.: Chemical Carcinogenesis. Little Brown and Company, Boston, Massachusetts. pp. 385 386, 1962

32) Lardy, H. A.; D. Johnson \& W. C. McMurray: Antibiotics as tools for metabolic studies. I. A survey of toxic antibiotics in respiratory phosphorylation and glycolytic systems. Arch. Biochem. Biophys. $78: 587 \sim 597,1958$

33) Watanabe, S.: Equimolecular reaction of showdomycin with thiols. J. Antibiotics $23: 313 \sim$ 314, 1970

34) Cramer, J. W.; J. A. Miller \& E. C. Miller: A new metabolic reaction observed in the rat with the carcinogen 2-acetylaminofluorene. J. Biol. Chem. $235: 885 \sim 888,1960$

35) Miller, J. A.; J. W. Cramer \& E. C. Miller: The N and ring hydroxylation of 2-acetylaminofluorene during carcinogenesis in the rat. Cancer Research $20: 950 \sim 962,1960$

36) Miller, E. C.; J. A. Miller \& H. A. HartmanN: N-Hydroxy-2-acetylaminofluorene: A metabolite of 2-acetylaminofluorene with increased carcinogenic activity in the rat. Cancer Research $21: 815 \sim 824,1961$

37) Mrller, J. A. \& E. C. Miller: The metabolic activation of carcinogenic aromatic amines and amides. Progress in Experimental Tumor Reseach 11:273 301, 1969 
38) Irving, C. C.: Enzymic N-hydroxylation of the carcinogen 2-acetylaminofluorene and the metabolism of N-hydroxy-2-acetylaminofluorene-9-C14 in vitro. J. Biol. Chem. 239: 1589 1596, 1964

39) Gutmann, H. R.; D. S. Leaf, L. Yost, R. E. Rydell \& C. C. Chen: Structure activity relationships of N-acylarylhydroxylamines in the rat. Cancer Research $30: 1485 \sim 1498,1970$

40) Clerici, E. \& G. Cudkovicz: Certain effects of $p$-dimethylaminoazobenzene, deficient diet and hypotonic media, on mitochondrial enzymes in experimental rat-liver carcinogenesis. J. Nat. Cancer Inst. $16: 1459 \sim 1470,1956$

41) Еммецот, P. \& C. J. Bos: The effect on thyroxine on the swelling of mitochondria isolated from normal and neoplastic livers. Exp. Cell Research $12: 191 \sim 195,1957$

42) Arcos, J. C.; G. W. Griffith \& R. W. Cunningham: Fine structural alterations in cell particles during chemical carcinogenesis. J. Biophys. \& Biochem. Cyto1. $7: 49 \sim 60,1960$

43) Arcos, J. C.; H. H. Gosch \& D. Zickafoose: Fine structural alterations in cell particles during chemical carcinogenesis. III. Selective action of hepatic carcinogens other than $3^{\prime}$-methyl-4dimethylaminoazobenzene on different types of mitochondrial swelling. Effect of stimulated liver growth. J. Biophys. \& Biochem. Cytol. $10: 23 \sim 36,1961$

44) Price, J. M.; E. C. Miller, J. A. Miller \& G. M. Weber: Studies on the intracellular composition of livers from rats fed various aminoazo dyes. I. 4-Aminoazobenzene, 4-dimethylaminoazobenzene, $4^{\prime}$-methyl and $3^{\prime}$-methy1-4-dimethylaminoazobenzene. Cancer Research 9 : $398 \sim 402,1949$

45) Price, J. M.; E. C. Miller, J. A. Miller \& G. M. Weber: Studies on the intracellular composition of livers from rats fed various aminoazo dyes. II. 3'-Methyl-2'-methyl and 2methyl-4-dimethylaminoazobenzene, 3-methyl-4-monomethylaminoazobenzene, and 4'-fluor-4dimethylaminoazobenzene. Cancer Research $10: 18 \sim 27,1950$

46) Streibich, M. J.; M. Shelton \& W. C. Schneider: Quantitative morphological studies on the livers and liver homogenates of rats fed 2-methyl- or $3^{\prime}$-methyl-4-dimethyl-aminoazobenzene. Cancer Research $13: 279 \sim 284,1953$

47) Schneider, W. C.: Intracellular distribution of enzymes. II. Distribution of succinic dehydrogenase, cytochrome oxidase, adenosinetriphosphatase and phosphorous compounds in normal rat liver and rat hepatomas. Cancer Research 6:685 690, 1946

48) Schneider, W. C. \& G. Hogeboom: Intracellular distribution of enzymes. VI. The distribution of succinoxidase and cytochrome oxidase activities in normal mouse liver and in mouse hepatoma. J. Nat. Cancer Inst. $10: 969 \sim 975,1950$

49) Wenner, C. E. \& S. Weinhouse: Metabolism of neoplastic tissue. IV. Diphosphopyridine nucleotide requirements for oxidations by mitochondria of neoplastic and non neoplastic tissues. Cancer Research $13: 21 \sim 26,1953$

50) Aisenberg, A.: The glycolysis and respiration of tumors. Academic Press, New York, 1961

51) Dulbecco, R.: Cell transformation by virusus. Science $166: 962 \sim 968,1969$

52) Baluda, M. A. \& D. P. NAYAK: DNA complementary to viral RNA in leukemic cells induced by avian myeloblastosis virus. Proc. Nat. Acad. Sci. U.S.A. $66: 329 \sim 336,1970$

53) NAss, M.M.K.: Mitochondrial DNA: Advances, problems, and goals. Science $165: 25 \sim 35,1969$

54) Nass, S.: The significance of the structural and functional similarities of bacteria and mitochondria. Int. Rev. Cytol. $25: 55 \sim 129,1969$

55) Cohen, S. S.: Are/were mitochondria and chloroplasts microorganisms? American Scientist $58: 281 \sim 289,1970$ 